\title{
SimCAL: a flexible tool to compute biochemical reaction similarity
}

\author{
Tadi Venkata Sivakumar ${ }^{1}$, Anirban Bhaduri', Rajasekhara Reddy Duvvuru Muni ${ }^{1}$, Jin Hwan Park ${ }^{2}$ \\ and Tae Yong $\mathrm{Kim}^{2 *}$
}

\begin{abstract}
Background: Computation of reaction similarity is a pre-requisite for several bioinformatics applications including enzyme identification for specific biochemical reactions, enzyme classification and mining for specific inhibitors. Reaction similarity is often assessed at either two levels: (i) comparison across all the constituent substrates and products of a reaction, reaction level similarity, (ii) comparison at the transformation center with various degrees of neighborhood, transformation level similarity. Existing reaction similarity computation tools are designed for specific applications and use different features and similarity measures. A single system integrating these diverse features enables comparison of the impact of different molecular properties on similarity score computation.

Results: To address these requirements, we present SimCAL, an integrated system to calculate reaction similarity with novel features and capability to perform comparative assessment. SimCAL provides reaction similarity computation at both whole reaction level and transformation level. Novel physicochemical features such as stereochemistry, mass, volume and charge are included in computing reaction fingerprint. Users can choose from four different fingerprint types and nine molecular similarity measures. Further, a comparative assessment of these features is also enabled. The performance of SimCAL is assessed on 3,688,122 reaction pairs with Enzyme Commission (EC) number from MetaCyc and achieved an area under the curve (AUC) of $>0.9$. In addition, SimCAL results showed strong correlation with state-of-the-art EC-BLAST and molecular signature based reaction similarity methods.

Conclusions: SimCAL is developed in java and is available as a standalone tool, with intuitive, user-friendly graphical interface and also as a console application. With its customizable feature selection and similarity calculations, it is expected to cater a wide audience interested in studying and analyzing biochemical reactions and metabolic networks.
\end{abstract}

Keywords: Reaction similarity, Transformation similarity, Similarity measures, Fingerprint

\section{Background}

Knowledge of biochemical reaction similarity is important for a wide range of biotechnological applications, such as, classification of enzymes [1-4], identification of missing enzymes in metabolic pathways $[5,6]$, identification of promiscuous enzymes in understanding the metabolic network evolution [7] and mine specific reaction substrates and the inhibitors [8-11]. Similarity between chemical reactions, referred to as reaction similarity, can be calculated at multiple levels: Transformation level

\footnotetext{
* Correspondence: ty76.kim@samsung.com

${ }^{2}$ Biomaterials Lab, Materials Center, Samsung Advanced Institute of

Technology, Gyeonggi-do 443803, South Korea

Full list of author information is available at the end of the article
}

similarity is computed by considering only the atoms and bonds that are undergoing transformation, at different degrees of neighborhood information [12]. Reaction level similarity considers molecular information of the entire substrates and products constituting a biochemical reaction [13]. Assessing reaction similarity as transformation level enables classification of enzyme function based on reaction mechanism [14-16]. Evaluating similarity at reaction level assists novel pathway engineering by identifying possible native target molecules in organisms and relevant possible enzymes that can catalyze novel steps [17-19].

Depending on the objective, reaction similarity computations rely on different feature representations to achieve required purposes. RxnFinder [20], a reaction 
search engine tool, uses Reaction Difference Fingerprint (RDF) for finding similar reactions. RDF is the difference between the union of features collected on substrate side and product side of a reaction. Unlike RDF, which is a fingerprint based representation of differences, RDM (reaction center, difference atom and matched atom) pattern [21] is a non-fingerprint based representation of transformation region. An extension of the RDM pattern is used in Metabolite and Reaction Inference based on Enzyme Specificities (MaRIboES) [22] for identifying specificity of an enzyme to catalyze a given metabolite or reaction. SimIndex (SI) and SimZyme [5] use two dimensional chemical fingerprints for computing chemical similarity for identifying new enzymatic connections in the metabolic networks. EC-BLAST [23] performs similarity searches using three different techniques, namely, bond changes $(\mathrm{BC})$, reaction centers (RC) and substructure similarity to search and compare enzymatic reactions. Enzyme promiscuity based on reaction similarity is studied using molecular graph descriptors (molsig) [24] . Numerous additional methods aiming to quantify molecular or reaction similarity are reported in literature $[25,26]$. From these perspectives it is evident that, computed reaction similarity results are dependent on factors such as the final objective, nature of data, choice of similarity measure and the fingerprint. Hence, obtaining consensus is challenging [27-30] (S1). Thus, it is imperative to customize the assessment in accordance with the application.

An integrated system enabling a combination of various similarity computation approaches along with a choice of features and comparative assessment of results would be of immense help. This article presents SimCAL, a robust tool that allows users to customize the reaction similarity assessment and evaluation in accordance with the desired application. The tool offers flexibility around the selection of different feature types and approaches to compute, compare reaction similarity.

\section{Implementation}

SimCAL is available both as a user-friendly graphical interface tool and a console application. It is developed in Java (ver 1.7) and uses chemoinformatics routines of Chemical Development Kit, CDK [31] for processing. The key modules of SimCAL are (i) parameter selection, (ii) process flow and (iii) analysis. These are described in Fig. 1. Parameter selection component enables the user to select different features along with the similarity type to be computed using the reaction data provided by the user. Process flow component, provides details of the steps involved in finding reaction similarity at the reaction and transformation levels. Analysis component provides user with several options to perform comparative assessments.

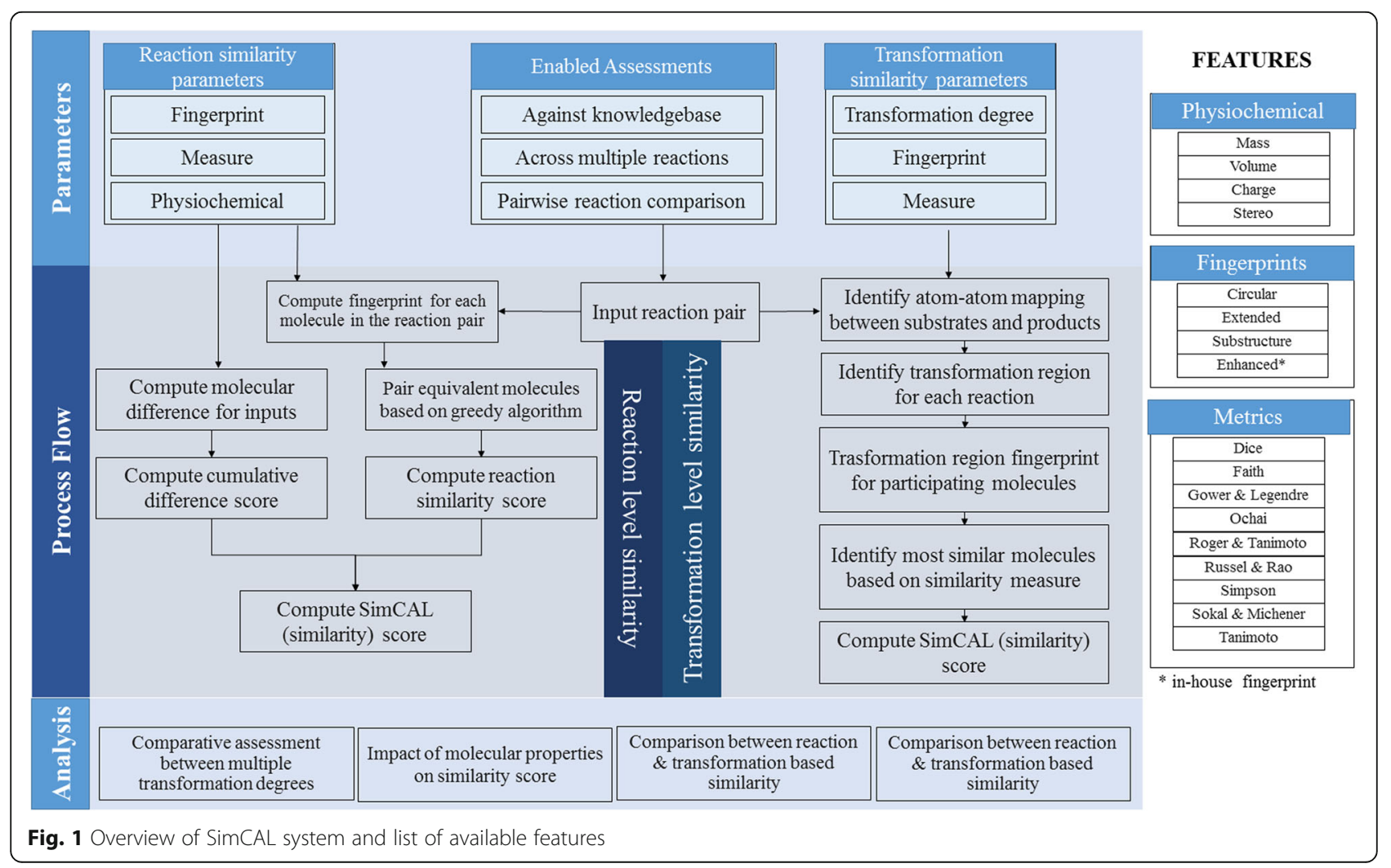




\section{Parameter selection}

Parameter selection module allows selection of different features and their representation that will be used to compute reaction similarity. User begins the analysis by selecting either or both of the reaction similarity types, namely, reaction level and transformation level. This is followed by the selection of any or all of the four fingerprints available in SimCAL, namely, (i) Circular, (ii) Extended, (iii) Substructure and (iv) Enhanced fingerprint. Details of these fingerprints are provided in Table 1. Enhanced fingerprint is an in-house developed improvisation of the extended fingerprint. In enhanced fingerprint, in addition to the molecular descriptors defined through specific binary bits, distinct signatures for charge and stereochemistry are encoded. Further, user can select any or all of the nine similarity measures for computing reaction similarity. The details of similarity measures, are provided in Table 2 . The similarity measure calculations are computed using four variables: $\boldsymbol{a}, \boldsymbol{b}, \boldsymbol{c}$ and $\boldsymbol{d}$. These variables capture the presence and absence of specific descriptors across two fingerprint vectors A and $B$ related to the two molecules under consideration. $\boldsymbol{a}$ is the count of set bits in both fingerprint of molecule A and B. $\boldsymbol{b}$ is the count of set bits in fingerprint of molecule A and not in B. $\boldsymbol{c}$ is the count of set bits in fingerprint of molecule B and not in A. $d$ is the count of unset bits in both the fingerprints of the molecules $\mathrm{A}$ and $\mathrm{B}$. The size of a fingerprint is given by $\boldsymbol{n}=(\boldsymbol{a}+\boldsymbol{b}+\boldsymbol{c}+\boldsymbol{d})$. The default selection measure used in SimCAL is Tanimoto. Reaction similarity calculations are further adjusted by considering variance of specific molecular properties such as mass, volume [32] and $\mathrm{pH}$ based charge calculations. Impact of the parameters (reaction similarity type, fingerprint, molecular properties, and measure) is highlighted using a simple dataset as discussed in Additional file 1: (S2, S3).

\section{Process flow}

SimCAL facilitates the computation of reaction similarity score based on transformation regions [25] and whole reaction level [7, 23, 33].
Table 2 List of binary similarity measures included in SimCAL

\begin{tabular}{llll}
\hline S. No. & Measure & Definition & Range \\
\hline 1. & Tanimoto & $\frac{a}{(a+b)+(a+c)-c}$ & {$[0-1]$} \\
2. & Dice & $\frac{2 a}{2 a+b+c}$ & {$[0-1]$} \\
3. & $\frac{a}{\sqrt{(a+b)}(a+c)}$ & {$[0-1]$} \\
4. & Schiai & $\frac{a}{\min (a+b, a+c)}$ & {$[0-1]$} \\
5. & Russell and Rao & $\frac{a}{a+b+c+d}$ & {$[0-1]$} \\
6. & Sokal and Michener & $\frac{a+d}{a+b+c+d}$ & {$[0-1]$} \\
7. & Faith & $\frac{a+0.5 d}{a+b+c+d}$ & {$[0-1]$} \\
8. & Gower and Legendre & $\frac{a+d}{a+0.5(b+c)+d}$ & {$[0-1]$} \\
9. & Roger and Tanimoto & $\frac{a+d}{a+2(b+c)+d}$ & {$[0-1]$} \\
\hline
\end{tabular}

The measures are in correspondence to [45]. $a$ is count of set bits in both fingerprint of both the molecules. $b$ is count of set bits in fingerprint of first molecule and not in second molecule. $c$ is count of set bits in fingerprint of second molecule and not in first molecule. $d$ is count of unset bits in both fingerprint of both the molecules. The size of the fingerprint is given by $n=(a+b+c+d)$

\section{Similarity score computation: Transformation region based}

Transformation region in a chemical reaction comprises of the reaction center (sets of atoms across the molecules undergoing bond rearrangement) and its neighborhood. The extent of the neighborhood defining the transformation region is captured through the transformation degree [34]. For example a transformation degree of one (which is default and can be defined by user) would comprise the reaction center and all atoms associated with the reaction center at one bond distance. The transformation region from a reaction is extracted based on the atom-atom mapping. The atom-atom mapping can either be provided by the user or calculated using reaction decoder tool (RDT) [35]. The extracted transformation region is further processed using the user selected fingerprint and measure to compute the reaction similarity using the reaction level similarity calculation procedure. Process outline for the computation of transformation similarity is shown in Fig. 2.

\section{Similarity score computation: Whole reaction level}

The computation of whole reaction level similarity considers all substrates and products in a reaction to the entirety. All constituent molecules in the reaction are

Table 1 List of four fingerprints available in SimCAL

\begin{tabular}{lll}
\hline S. No. & Name & Description \\
\hline 1. & Circular Fingerprint & Circular fingerprint is based on CDK's [31] circular fingerprinter and is functionally equivalent to ECFP-2 [43] \\
2. & Extended Fingerprint & $\begin{array}{l}\text { Functionally equivalent to ExtendedFingerprinter of CDK [31]. This fingerprint is unique from the standard form } \\
\text { since it accounts for ring systems. Default length size is 1024 bits. }\end{array}$ \\
3. & Substructure Fingerprint & $\begin{array}{l}\text { This is a structural key type fingerprint which considers assessment of } 307 \text { different substructures and is based on } \\
\text { KlekotaRothFingerprinter [44] in CDK. }\end{array}$ \\
Anhanced Fingerprint & $\begin{array}{l}\text { An in-house developed improvised extended fingerprint which accounts for stereochemistryand charges on } \\
\text { molecules. }\end{array}$ \\
\hline
\end{tabular}




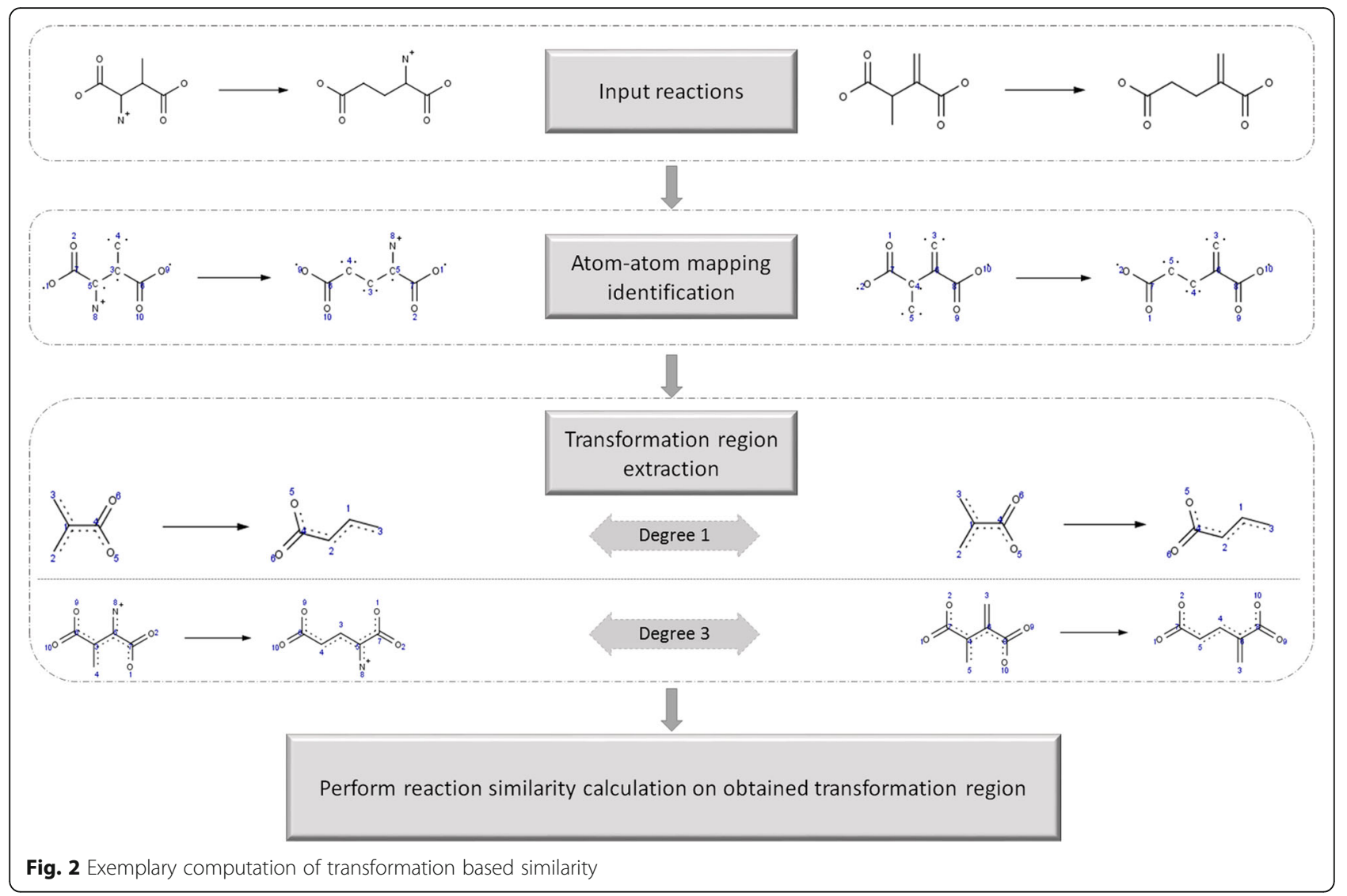

represented in a reaction fingerprint vector. The fingerprints or molecule descriptors vary for different fingerprint methods. This conversion is performed for each input reaction. A greedy algorithm is used to pair molecules across the reactions [13]. The objective of the pairing is to maximize user selected similarity measure. The reaction similarity score is the average of the molecular similarity [13] computed for all equivalent pairs of molecules. Any unpaired molecules are dropped from computations. A schematic of the processing is depicted in Fig. 3.

\section{Similarity computation: Molecular property correction}

A general constraint of reaction similarity calculation methods is that they do not consider deviation of physicochemical attributes of the constituent molecules in the reaction pair. This can result in erroneous computation of similarity scores. Changes in $\mathrm{pH}$ influences the charge of constituent molecules in a reaction, affecting its transformation feasibility. SimCAL provides flexible options for considering four molecular properties viz., stereochemistry, charge, mass and volume in the computation of similarity between reactions. Stereochemistry and molecular charge of constituent molecules of a reaction are assessed using the circular fingerprinter [31] and an in-house developed enhanced fingerprint. Since they are represented as bits within the fingerprint vector, their impact is accounted for while computing the reaction similarity score using a selected measure (Fig. 3). The impact of environment such as $\mathrm{pH}$ on a chemical transformation is well documented [36]. SimCAL accepts a user defined $\mathrm{pH}$ value (default 7) to compute theoretical pKa of input molecules [37] and report the charge on the constituent molecules. Based on the reported charge distribution on constituent molecules, the in-house developed enhanced fingerprint is then used to compute reaction similarity.

SimCAL computes the molecular mass and the molecular volume of the constituents of the reaction as implemented in CDK. The variability associated with mass and volume between the paired molecular entities are computed using a generalized Jaccard distance [38]. The computed average Jaccard distance along with the reaction fingerprint based similarity score is used to compute the final reactions similarity (Eq. 1).

$$
R_{s}=R_{f} / 1+J_{\text {dist }}
$$

where $R_{s}=$ Reaction similarity score, $R_{f}=$ Reaction similarity score based on fingerprint and $J_{\text {dist }}=$ Variation of molecular property obtained through Jaccard distance $J_{\text {dist }}$ is the average Jaccard distance, Eq. (2). This is obtained from 


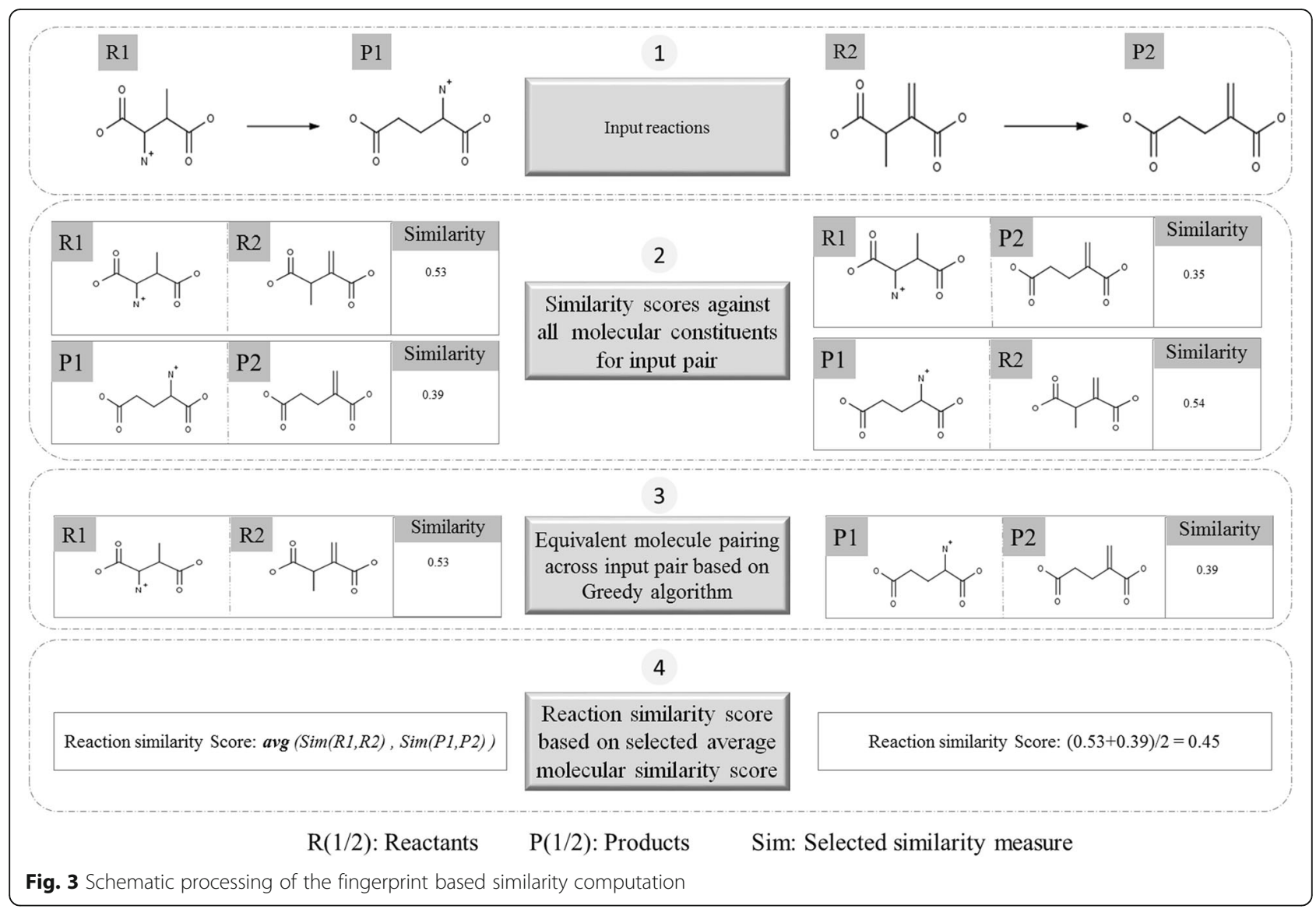

the generalized Jaccard score $\left(J_{s}\right)$, Eq. (3) for $N$ paired molecules in the under study reaction pairs. Each equivalent pair of molecules is represented by $a$, and $b$. The Jaccard distance $J_{\text {dist }}$ may be computed for the selected properties based on the selection of a user (mass, volume or both).

$$
\begin{aligned}
& J_{\text {dist }}=1-\sum\left(J_{s}\right) /{ }_{N} \\
& J_{s}=\min (a, b) / \max (a, b)
\end{aligned}
$$

\section{Analysis}

The analysis enables user to further customize and assess similarity calculations through comparative assessment. SimCAL provides three types of comparative assessment techniques: (i) transformation degree comparative assessment, (ii) fingerprint comparative assessment and (iii) similarity measure comparative assessment. Transformation degree based assessment provides transformation level based similarity by considering different degrees of user selected neighborhood length. Fingerprint based comparative assessment can be used to compare the results obtained from different fingerprints the user has selected. To compare reaction similarity results of chosen molecular similarity measures, similarity measure comparative assessment can be used. All these comparative assessments can be performed at both reaction level as well as transformation level. Once a simulation is completed on user provided data, SimCAL provides a unique feature to either select entire set of reactions or a subset of results to re-evaluate them using other parameter selection.

\section{Results \& discussion}

SimCAL feature evaluation

As per the four digit Enzyme Commission (EC) nomenclature, two reactions are said to be similar if the enzymes catalyzing those reactions are identical up to the 3rd level (sub-subclass) [39].Reaction pairs catalyzed by enzymes having EC number until the first 3 digits were classified similar (true positive), while others where annotated as not similar (true negative). Using this hypothesis, we evaluated the performance of SimCAL to compute reaction similarity with the following parameters:

- Transformation similarity (degree 1 )

- Reaction similarity based on extended fingerprint

- Reaction similarity based on enhanced fingerprint (considers charge and stereo-centers) 
- Reaction similarity based on enhanced fingerprint and molecular properties (mass and volume)

The dataset comprised of 3,688,122 reaction pairs obtained by pairing (all-against-all) reactions from MetaCyc [40] within each EC class. The prediction performance was accessed using receiver operator characteristic (ROC) as implemented in the ROCR package [41]. The Area under the curve (AUC), that estimates the robustness of the method, calculated for the above four parameters are as follows: $0.92,0.89,0.90$ and 0.90 . The performance of different ROC properties trends against a threshold score (cutoff) is plotted in Fig. 4b. The prediction of the accuracy of the methods are provided in Fig. 4a. The accuracy of reaction similarity based on enhanced fingerprint and molecular properties has the best accuracy, which also has higher precision value as shown in Fig. 4b. The recall plot on the other hand suggests that the transformation similarity based approach performs better. The ROC experiments suggest that the reaction similarity obtained by using enhanced fingerprints and molecular properties outperforms other approaches.

\section{Benchmarking over existing methods}

Further SimCAL's performance is benchmarked against two existing methods EC-BLAST [23] and the molecular signature based reaction similarity method [24]. For benchmarking study we consider the molecular signature based reaction chemical similarity method [24] (with $\mathrm{h}$ set to 4) and all the three approaches provided in EC-BLAST [23]. Along with these, three features considered from SimCAL are transformation level similarity with degree 1 , reaction level similarity using extended fingerprint and enhanced fingerprint along with molecular property variance. It should be noted that SimCAL uses bit based fingerprints whereas the two tools against which it is compared consider count based fingerprint for their assessment.

The same dataset used for SimCAL feature evaluation is used for benchmarking as well. Pearson correlations of the results between approaches are summarized in
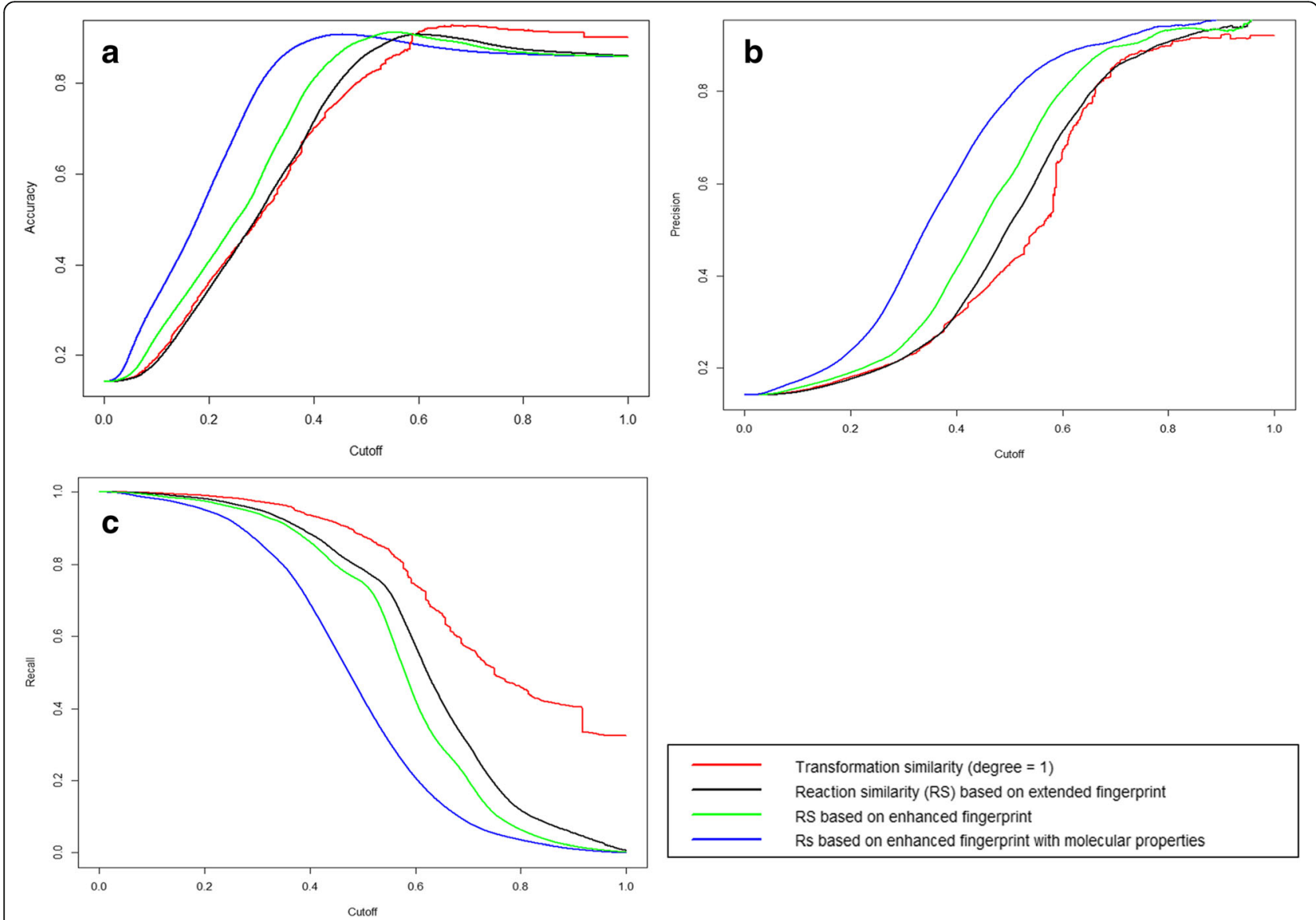

Fig. 4 Receiver operating curves (ROC) for various approaches. a Reports the dependency of accuracy of predicting similar and non-similar reactions with cutoff (threshold) using the various approaches. $\mathbf{b}$ Reports the dependency of precision of predicting similar and non-similar reactions with cutoff (threshold) using the various approaches. $\mathbf{c}$ Reports the dependency of recall (true predictive rate) of predicting similar and non-similar reactions with cutoff (threshold) using the various approaches 


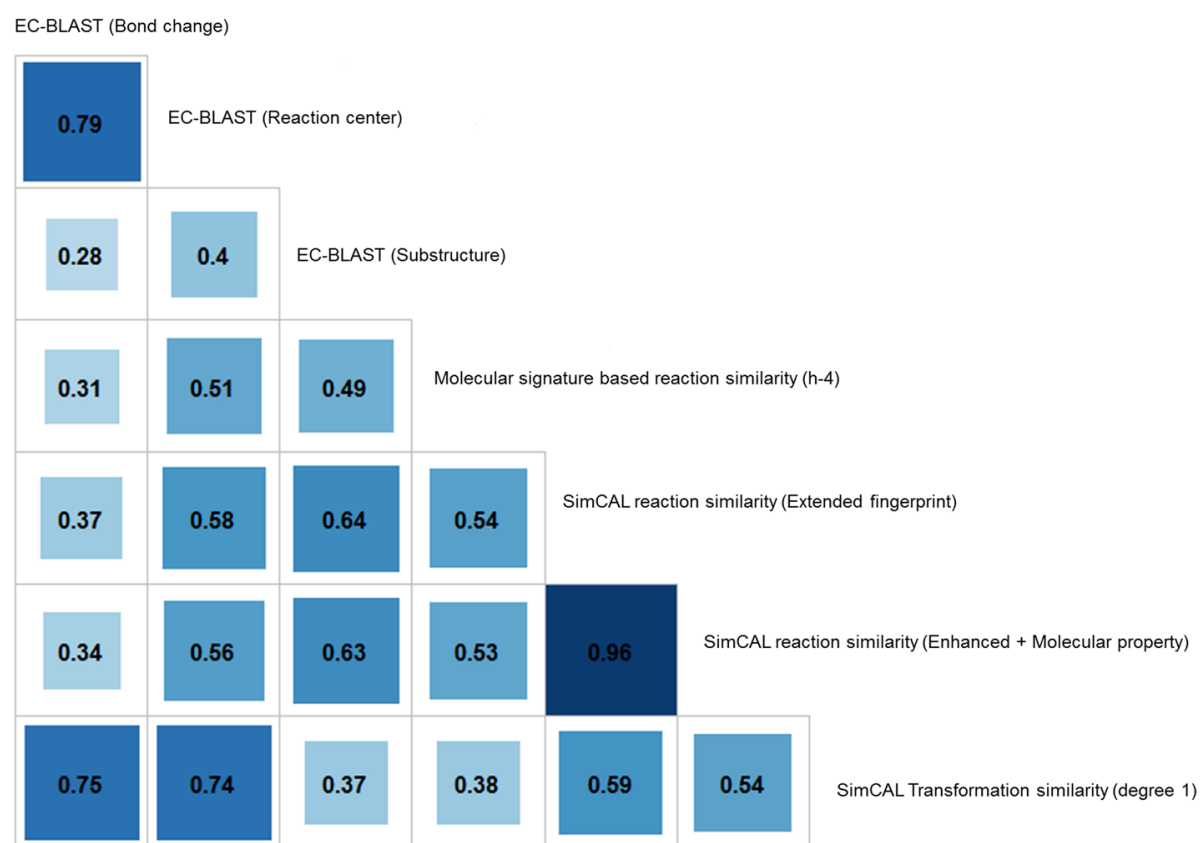

Fig. 5 Correlation matrix across the various approaches within SimCAL and 3 approaches of EC-BLAST and the molecular signature based chemical similarity method

Fig. 5. The intensity of color in the box is directly proportional to the correlation between any two methods under consideration. The correlation analysis shows that results obtained by EC-BLAST (bond change), EC-BLAST (reaction center) and SimCAL transformation similarity are well correlated among each other with minimum value of 0.74 and maximum of 0.79 . SimCAL (extended fingerprint) (0.54) is correlated slightly higher to the molecular signature based reaction similarity than EC-BLAST (reaction center) (0.51). Both SimCAL (extended fingerprint) and SimCAL (enhanced fingerprint + molecular property) show a very high correlation of 0.96 . This is due to the fact that the dataset contains very few reactions, catalyzed by the same enzyme class up to 3rd digit have differences in stereo or charge or molecular property variance. It was observed that the approaches at a large scale shares moderate to strong correlation [42].

\section{Conclusion}

The identification of reaction similarity has a growing range of applications in biochemistry. SimCAL, the integrated tool presented here, enables reaction similarity computation at different levels with a wide choice of feature selection and comparative assessment of final results. The reaction similarity computation is further enhanced by using additional molecular properties, stereo and charge specific information. It is believed that the tool will cater to a wide audience in the field of biochemistry and metabolic engineering.

\section{Availability and requirements}

\section{Project Name: SimCal.}

Project home page: https://sourceforge.net/projects/ simcal/

Operating systems: Windows, Linux and Mac.

Programming language: Java.

Other requirements: Java 1.7 or higher.

License: LGPL.

Data generated and analyzed during the current research is available in the supplementary data files, along with the R scripts.

\section{Additional file}

Additional file 1: Supplementary material. (DOCX 1098 kb)

Abbreviations

AUC: Area under the curve; CDK: Chemistry development kit; RDT: Reaction decoder tool; ROC: Receiver operator characteristics

\section{Acknowledgements}

Authors acknowledge support from Samsung Advanced Institute of Technology.

\section{Funding}

The current work was supported entirely by Samsung Advanced Institute of Technology.

Authors' contributions

$T V, A B, T K$, JP conceived the idea. TV, $A B$ has contributed to the data collection. $T V$ was the lead in design and implementation of the system. AB, RRDV, JP, TK contributed to the experimental design and analysis. TV, AB, RRDV, TK drafted the manuscript. All the authors have approved the manuscript. 


\section{Ethics approval and consent to participate}

Not applicable.

\section{Consent for publication}

Not applicable.

\section{Competing interests}

The authors declare that they have no competing interests.

\section{Publisher's Note}

Springer Nature remains neutral with regard to jurisdictional claims in published maps and institutional affiliations.

\section{Author details}

'Bioinformatics Lab, Samsung Advanced Institute of Technology, Bangalore 560037, India. ²Biomaterials Lab, Materials Center, Samsung Advanced Institute of Technology, Gyeonggi-do 443803, South Korea.

\section{Received: 18 August 2017 Accepted: 14 June 2018}

\section{Published online: 03 July 2018}

\section{References}

1. Egelhofer V, Schomburg I, Schomburg D. Automatic assignment of EC numbers. PLoS Comput Biol. 2010;6:e1000661.

2. Hu QN, Zhu H, Li X, Zhang M, Deng Z, Yang X, et al. Assignment of EC numbers to enzymatic reactions with reaction difference fingerprints. PLoS One. 2012;7:e52901.

3. Dönertaş HM, Martínez Cuesta S, Rahman SA, Thornton JM. Characterising complex enzyme reaction data. PLoS One. 2016;11:e0147952.

4. Nath N, Mitchell JB. Is EC class predictable from reaction mechanism? BMC Bioinformatics. 2012;13:60.

5. Pertusi DA, Stine AE, Broadbelt LJ, Tyo KEJ. Efficient searching and annotation of metabolic networks using chemical similarity. Bioinformatics [Internet]. 2015;31:1016-1024. Available from: http://www.ncbi.nlm.nih.gov/ pubmed/25417203

6. Tabei Y, Yamanishi Y, Kotera M. Simultaneous prediction of enzyme orthologs from chemical transformation patterns for de novo metabolic pathway reconstruction. Bioinformatics. 2016;32:i278-87.

7. Carbonell P, Lecointre G, Faulon J-L. Origins of specificity and promiscuity in metabolic networks. J Biol Chem. 2011;286:43994-4004. Available from: http://www.ncbi.nlm.nih.gov/pubmed/22052908

8. Rose JR, Gasteiger J. HORACE: An automatic system for the hierarchical classification of chemical reactions. J Chem Inf Model. 1994:34:74-90. Available from: http://pubs.acs.org/cgi-bin/doilookup/?10.1021/ci00017a010

9. Xia J, Tilahun EL, Reid TE, Zhang L, Wang XS. Benchmarking methods and data sets for ligand enrichment assessment in virtual screening. Methods. 2015;71:146-57.

10. Ripphausen P, Wassermann AM, Bajorath J. REPROVIS-DB: a benchmark system for ligand-based virtual screening derived from reproducible prospective applications. J Chem Inf Model. 2011;51:2467-73.

11. Fukunishi Y. Structure-based drug screening and ligand-based drug screening with machine learning. Comb Chem High Throughput Screen 2009;12:397-408

12. Yamanishi Y, Hattori M, Kotera M, Goto S, Kanehisa M. E-zyme: predicting potential EC numbers from the chemical transformation pattern of substrate-product pairs. Bioinformatics. 2009;25:1179-86. Available from: https://academic.oup.com/bioinformatics/article-lookup/doi/10.1093/ bioinformatics/btp223

13. Giri V, Sivakumar TV, Cho KM, Kim TY, Bhaduri A. RxnSim: a tool to compare biochemical reactions. Bioinformatics. 2015;31:3712-4.

14. Holliday GL, Andreini C, Fischer JD, Rahman SA, Almonacid DE, Williams ST, et al. MACiE: exploring the diversity of biochemical reactions. Nucleic Acids Res. 2012;40:D783-9. Available from: https://academic.oup.com/nar/articlelookup/doi/10.1093/nar/gkr799

15. Almonacid D, Babbitt P. Toward mechanistic classification of enzyme functions. Curr Opin Chem Biol. 2011;15:435-42.

16. O'Boyle NM, Holliday GL, Almonacid DE, Mitchell JB. Using reaction mechanism to measure enzyme similarity. J Mol Biol. 2007;368:1484-99.

17. Liu M, Bienfait B, Sacher O, Gasteiger J, Siezen RJ, Nauta A, et al. Combining chemoinformatics with bioinformatics: in silico prediction of bacterial flavor- forming pathways by a chemical systems biology approach reverse pathway engineering. PLoS One. 2014:9:e84769.

18. Christ CD, Zentgraf M, Kriegl JM. Mining electronic laboratory notebooks: analysis, retrosynthesis, and reaction based enumeration. J Chem Inf Model. 2012:52:1745-56.

19. Gasteiger J. Modeling chemical reactions for drug design. J Comput Aided Mol Des. 2007;21:33-52

20. Hu Q-N, Deng Z, Hu H, Cao D-S, Liang Y-Z. RxnFinder: biochemical reaction search engines using molecular structures, molecular fragments and reaction similarity. Bioinformatics. 2011;27:2465-7.

21. Oh M, Yamada T, Hattori M, Goto S, Kanehisa M. Systematic Analysis of Enzyme-Catalyzed Reaction Patterns and Prediction of Microbial Biodegradation Pathways. J Chem Inf Model. 2007;47:1702-12. Available from: http://pubs.acs.org/doi/abs/10.1021/ci700006f

22. DeGroot MJL, Van Berlo RJP, Van Winden WA, Verheijen PJT, Reinders MJT, De Ridder D. Metabolite and reaction inference based on enzyme specificities. Bioinformatics. 2009;25:2975-82.

23. Rahman SA, Cuesta SM, Furnham N, Holliday GL, Thornton JM. EC-BLAST: a tool to automatically search and compare enzyme reactions. Nat Methods. 2014;11:171-4. Available from: http://www.ncbi.nlm.nih.gov/pubmed/ 24412978

24. Carbonell P, Carlsson L, Faulon J. Stereo signature molecular descriptor. J Chem Inf Model. 2013:53:887-97.

25. Schneider N, Lowe DM, Sayle RA, Landrum GA. Development of a nove fingerprint for chemical reactions and its application to large-scale reaction classification and similarity. J Chem Inf Model. 2015;55:39-53.

26. Stumpfe D, Bajorath J. Similarity searching. WIRES Comput Mol Sci. 2011;1: 260-82.

27. Al Khalifa A, Haranczyk M, Holliday J. Comparison of nonbinary similarity coefficients for similarity searching, clustering and compound selection. J Chem Inf Model. 2009;49:1193-201.

28. Todeschini R, Consonni V, Xiang H, Holliday J, Buscema M, W P. Similarity coefficients for binary chemoinformatics data: overview and extended comparison using simulated and real data sets. J Chem Inf Model. 2012:52: 2884-901

29. Rupp M, Schneider P, Schneider G. Distance phenomena in highdimensional chemical descriptor spaces: consequences for similarity-based approaches. J Comput Chem. 2009:30:2285-96.

30. Willett P. Similarity-based approaches to virtual screening. Biochem Soc Trans. 2003:31:603-6.

31. Steinbeck C, Hoppe C, Kuhn S, Floris M, Guha R, Willighagen EL. Recent developments of the chemistry development kit (CDK) - an opensource java library for chemo- and bioinformatics. Curr Pharm Des 2006;12:2111-20.

32. Zhao $\mathrm{YH}$, Abraham MH, Zissimos $\mathrm{AM}$. Fast calculation of van der Waals volume as a sum of atomic and bond contributions and its application to drug compounds. J Org Chem. 2003:68:7368-73.

33. Patel H, Bodkin MJ, Chen B, Gillet VJ. Knowledge-based approach to de novo design using reaction vectors. J Chem Inf Model. 2009:49:1163-84.

34. Sivakumar T, Giri V, Park J, Kim TY, Bhaduri A. ReactPRED: a tool to predict and analyze biochemical reactions. Bioi2. 2016; https://doi.org/10.1093/ bioinformatics/btw491.

35. Rahman SA, Torrance G, Baldacci L, Martínez Cuesta S, Fenninger F, Gopal N, et al. Reaction Decoder Tool (RDT): extracting features from chemical reactions.Bioinformatics. 2016;32:2065-6.

36. Pfeiffer J. Enzymes, the physics and chemistry of life. NY: Simon and Schuster; 1954. p. 171-3.

37. Lee AC, Yu J-Y, Crippen GM. pKa prediction of monoprotic small molecules the SMARTS way. J Chem Inf Model. 2008:48:2042-53.

38. Sepkoski J. Quantified coefficients of association and measurement of similarity. Math Geol. 1974;6:131-52.

39. Tipton KF. Nomenclature Committee of the International Union of Biochemistry and Molecular Biology (NC-IUBMB). Enzyme nomenclature. Recommendations 1992. Supplement: corrections and additions. Eur J Biochem. England; 1994;223:1-5

40. Caspi R, Billington R, Ferrer L, Foerster $H$, Fulcher $C$, Keseler I, et al. The MetaCyc database of metabolic pathways and enzymes and the BioCyc collection of pathway/genome databases. Nucleic Acids Res. 2016:44: D471-80.

41. Sing $T$, Sander $O$, Beerenwinkel N, Lengauer T. ROCR: visualizing classifier performance in R. Bioinformatics. 2005;21:3940-1. 
42. Ratner $B$. The correlation coefficient: its values range between $+1 /-1$, or do they? J Targeting Meas Anal Mark. 2009;17:139-42.

43. Rogers D, Hahn M. Extended-connectivity fingerprints. J Chem Inf Model. 2010;50:742-54.

44. Klekota J, Roth FP. Chemical substructures that enrich for biological activity. Bioinformatics. 2008;24:2518-25. Available from: http://www.ncbi.nlm.nih. gov/pubmed/18784118

45. Choi S, Cha S, Tappert C. A survey of binary similarity and distance measures. J Syst Cybern Informatics. 2010;8:43-8.

Ready to submit your research? Choose BMC and benefit from:

- fast, convenient online submission

- thorough peer review by experienced researchers in your field

- rapid publication on acceptance

- support for research data, including large and complex data types

- gold Open Access which fosters wider collaboration and increased citations

- maximum visibility for your research: over $100 \mathrm{M}$ website views per year 